The Astrophysical JournaL, 649: L000-L000, 2006 SEPTEMBER 20

Preprint typeset using $\mathrm{LT}_{\mathrm{E}} \mathrm{X}$ style emulateapj v. 08/13/06

\title{
EVERSHED CLOUDS AS PRECURSORS OF MOVING MAGNETIC FEATURES AROUND SUNSPOTS
}

\author{
D. Cabrera Solana ${ }^{1}$, L.R. Bellot Rubio ${ }^{1}$, C. BeCK $^{2}$, And J.C. Del Toro Iniesta ${ }^{1}$ \\ Received 2006 May 17; accepted 2006 August 7
}

\begin{abstract}
The relation between the Evershed flow and moving magnetic features (MMFs) is studied using highcadence, simultaneous spectropolarimetric measurements of a sunspot in visible $(630.2 \mathrm{~nm})$ and near-infrared $(1565 \mathrm{~nm})$ lines. Doppler velocities, magnetograms, and total linear polarization maps are calculated from the observed Stokes profiles. We follow the temporal evolution of two Evershed clouds that move radially outward along the same penumbral filament. Eventually, the clouds cross the visible border of the spot and enter the moat region, where they become MMFs. The flux patch farther from the sunspot has the same polarity of the spot, while the MMF closer to it has opposite polarity and exhibits abnormal circular polarization profiles. Our results provide strong evidence that at least some MMFs are the continuation of the penumbral Evershed flow into the moat. This, in turn, suggests that MMFs are magnetically connected to sunspots.
\end{abstract}

Subject headings: polarization - Sun: magnetic fields - Sun: photosphere - sunspots

\section{INTRODUCTION}

Moving magnetic features (MMFs) are small flux concentrations observed in the moat surrounding sunspots. They were discovered by Sheeley (1969). Not surprisingly, a connection between MMFs and the magnetic field of sunspots was proposed a few years later by Harvey \& Harvey (1973). The phenomenological properties of MMFs are relatively well know by now (Shine \& Title 2001), but their origin and magnetic structure remain a mystery. The situation is more favorable in the case of sunspots (Solanki 2003). According to current views, sunspot penumbrae are formed by at least two magnetic components with different field inclinations. The more horizontal component drives the Evershed flow from the inner to the outer penumbra, where it partly dives down below the photosphere or continues into the canopy. The Evershed flow is not steady. Shine et al. (1994), Rimmele (1994), and Rouppe van der Voort (2003) have demonstrated the existence of velocity packages, called "Evershed clouds", that propagate radially outward and show an irregular repetitive behavior on a timescale of the order of 10-15 minutes. Evershed clouds evolve and remain coherent until they go through the outer penumbral border, where they seem to vanish.

Some investigators have pointed out the possible link between MMFs and the penumbral component carrying the Evershed flow (Martínez Pillet 2002; Schlichenmaier 2002; Thomas et al. 2002). In fact, recent observations seem to support this idea. Using Michelson Doppler Imager magnetograms, Sainz Dalda \& Martínez Pillet (2005) discovered magnetic filaments extending from the midpenumbra into the moat. Several bipolar MMFs were observed to leave the sunspot following the paths traced by these filaments. Zhang et al. (2003) and Penn et al. (2006) also detected the passage of MMFs through the outer penumbral boundary and their subsequent evolution across the moat. Bonet et al. (2004) applied a phase diversity restoration to G-band filtergrams of a sunspot. They concluded that most G-band bright points, which are associated with MMFs (Beck et al. 2006), are born close to the continuation of dark penumbral filaments. Hence,

\footnotetext{
${ }^{1}$ Instituto de Astrofísica de Andalucía (CSIC), Apdo. de Correos 3004 E-18080 Granada, Spain; dcabrera@iaa.es; lbellot@iaa.es; jti@iaa.es

${ }^{2}$ Kiepenheuer-Institut für Sonnenphysik, Schöneckstr. 6, 79104, Freiburg, Germany; cbeck@kis.uni-freiburg.de
}

they pointed out the possible relationship between G-band bright points (or MMFs) and the more horizontal component of the penumbra. Finally, Kubo (2005) analyzed Advanced Stokes Polarimeter measurements. He found significant correspondence between the magnetic field of MMFs and the penumbral uncombed structure.

In this Letter, the connection between the Evershed flow and MMFs is established. Using high resolution spectropolarimetric measurements in two different spectral ranges, we track the evolution of Evershed clouds from the midpenumbra to well outside the outer penumbral boundary, demonstrating that they become MMFs once they leave the sunspot.

\section{OBSERVATIONS AND DATA ANALYSIS}

NOAA AR 10781 was observed on 2005 June 30 from 8:47 to 11:23 UT with the German Vacuum Tower Telescope (VTT) of Observatorio del Teide (Tenerife, Spain) at an heliocentric angle of $43^{\circ}$. The Tenerife Infrared Polarimeter (TIP; Martínez Pillet et al. 1999) and the POlarimetric LIttrow Spectrograph (POLIS; Beck et al. 2005a) were operated simultaneously to record the full Stokes profiles of the Fe I lines at $1564.8,1565.2,630.15$, and $630.25 \mathrm{~nm}$. The slit width was $0 . " 36$ for TIP and $0 . " 18$ for POLIS. We performed rapid scans of a small portion of the center-side penumbra of the spot, including the adjacent moat region (see Figure 1). The scan step was $0 . " 2$ for a total of 20 steps $\left(4^{\prime \prime}\right)$. The integration time was $10.5 \mathrm{~s}$ per slit position, resulting in a cadence of 3.9 minutes. We performed 40 repetitions of the scan. During the observations, the Kiepenheuer Adaptive Optics System (Soltau et al. 2002) was used to reduce image motion and blurring. This, together with excellent seeing conditions, allowed us to reach a spatial resolution of about $0.16-0.7$. The data sets have been coaligned by cross-correlation using the 0 ." $175 \times 0$ " 175 TIP pixel as a reference (Beck et al. 2006).

After correcting the measurements for instrumental polarization (Beck et al. 2005b), residual crosstalk is estimated to be on the order of $10^{-3}$ in units of the continuum intensity. The velocity scale for TIP has been set calculating a mean quiet Sun intensity profile. The wavelengths of the line cores in the mean profile, corrected for convective blueshifts (Borrero \& Bellot Rubio 2002), represent the position of zero velocity. For POLIS, we have used the teluric $\mathrm{O}_{2}$ lines to perform an absolute velocity calibration for each repetition of the 


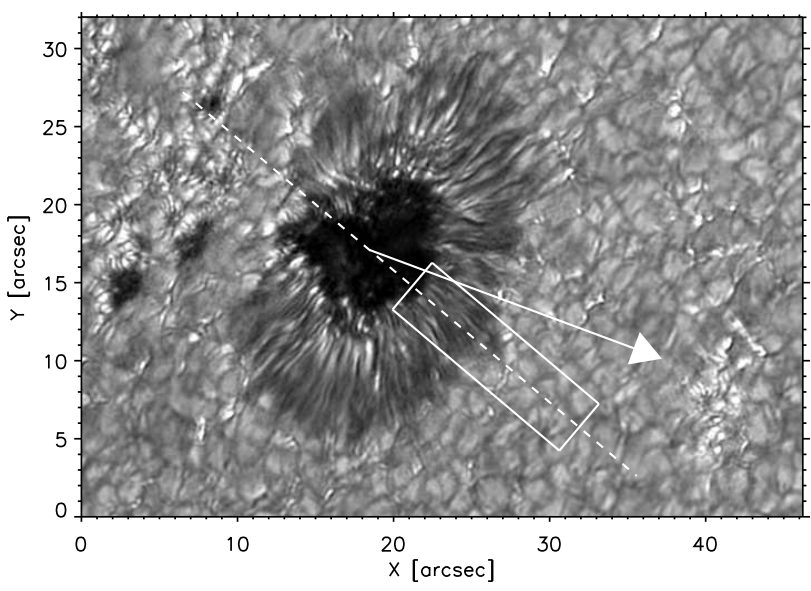

FIG. 1.- Speckle-reconstructed G-band filtergram of AR 10781 taken at the Dutch Open Telescope (DOT) on 2005 June 30, 9:34 UT. The box shows the fraction of the center-side penumbra and adjacent moat scanned by TIP and POLIS. The arrow marks the direction toward disk center.

scan in the same way as Martínez Pillet et al. (1997).

Line-of-sight (LOS) velocities and magnetograms have been calculated from the observed circular polarization profiles $V(\lambda)$. LOS velocities are estimated from the Doppler shifts of the Stokes $V$ zero-crossing wavelengths. These velocities are representative of the magnetic atmosphere inside the resolution element. Additionally, we construct magnetograms, $M$, as (minus) the Stokes $V$ signal of Fe I 630.25 $\mathrm{nm}$ at $\Delta \lambda=+10 \mathrm{pm}$ from line center. $M$ provides a rough estimate of the longitudinal magnetic flux. The total linear polarization, $L=\sum_{i}\left[Q\left(\lambda_{i}\right)^{2}+U\left(\lambda_{i}\right)^{2}\right]^{1 / 2}$, is also calculated. For weak lines, $L$ is proportional to $\sin ^{2} \gamma$ if the magnetic field orientation remains constant along the LOS. Hence, the larger the value of $L$, the greater the field inclination to the LOS.

\section{RESULTS}

In Figure 2 we show the temporal evolution of the centerside penumbra and moat region studied in this work. The first panel displays the continuum intensity at $1565 \mathrm{~nm}$. The next two panels show the magnetograms derived from Fe I $630.25 \mathrm{~nm}$ and the total linear polarization of the Fe I line at $1564.85 \mathrm{~nm}$. The last two panels display the LOS velocities calculated from the Stokes $V$ zero-crossing shifts of Fe I 1565.28 and Fe I $630.25 \mathrm{~nm}$. The velocity is computed only for pixels exhibiting normal two-lobed $V$ profiles with amplitudes greater than $0.2 \%$ for Fe I $630.25 \mathrm{~nm}$ and $0.5 \%$ for Fe I $1564.85 \mathrm{~nm}$.

During the time sequence, two opposite-polarity flux patches are observed to move away from the spot in the moat region (cf. the area enclosed by the green box in the magnetograms of Figure 2]. We have outlined them with pink and blue contours, and will refer to them as features A and B, respectively. The properties of this pair are similar to those of bipolar MMFs: the separation between the two polarities is around $2^{\prime \prime}$ (Zhang et al. 2003), the magnetic patch having the same polarity as the sunspot is located farther from the spot (Lee 1992; Yurchyshyn et al. 2001; Zhang et al. 2003), and the average proper motion, corrected for the viewing angle, is around $0.5 \mathrm{~km} \mathrm{~s}^{-1}$ (Nye et al. 1984; Zhang et al. 2003). Hence, features A and B could be classified as a bipolar MMF or, according to Shine \& Title (2001), as a type I MMF. However, from the magnetogram data alone we cannot rule out the possibility that A and B are two unrelated MMFs of type II and III. In any case, the second and third panels of Figure 2 demonstrate that the two flux patches possess different field inclinations. While feature A shows up in both $L$ and $M$, feature $\mathrm{B}$ is observed mainly in $M$. This indicates that $\mathrm{A}$ has more inclined magnetic fields to the LOS than B.

Our high-cadence observations make it possible to investigate the formation of these MMFs. Going backwards in time, one can see in the velocity maps that the origin of feature $\mathrm{A}$ is an Evershed cloud that shows up in the midpenumbra at $t=7.8$ minutes. The cloud is detected as a region of increased velocity signals, with maximum blueshifts of $3.2 \mathrm{~km} \mathrm{~s}^{-1}$ in Fe I $630.25 \mathrm{~nm}$ and $4.1 \mathrm{~km} \mathrm{~s}^{-1}$ in Fe I $1565.28 \mathrm{~nm}$. Initially its spatial extent is $750 \mathrm{~km}$, but as time goes on it becomes longer toward the outer penumbral boundary, reaching $2250 \mathrm{~km}$ at $t=31.3$ minutes. Near the sunspot edge, the cloud adopts a roundish shape ( $t=35.2-43.1$ minutes). The third panel of Figure 2 demonstrates that this structure moves radially outward following a penumbral filament with larger values of the linear polarization (that is, larger inclinations of the magnetic field to the LOS) than its surroundings. Its average propagation speed inside the penumbra is $\sim 1.7 \mathrm{~km} \mathrm{~s}^{-1}$. The Evershed cloud crosses the penumbral boundary between $t=47.0$ and $t=50.9$ minutes. Outside the spot, it is observed both in the velocity maps and in the magnetograms as a negative polarity MMF.

The precursor of feature B is another Evershed cloud first detected at $t=0$ minutes in the midpenumbra. It propagates along the very same penumbral filament as feature A, crossing the outer boundary of the spot at $t=11.7$ minutes. The cloud moves with an average speed of $3.3 \mathrm{~km} \mathrm{~s}^{-1}$ inside the penumbra and exhibits maximum blueshifts of $3.2 \mathrm{~km} \mathrm{~s}^{-1}$ (Fe I $630.25 \mathrm{~nm}$ ) and $4.4 \mathrm{~km} \mathrm{~s}^{-1}$ (Fe I $1565.28 \mathrm{~nm}$ ). Once in the moat region, it is still observed as a velocity structure until $t=27.4$ minutes, and then it shows up in the magnetograms as a positive polarity MMF.

\section{DISCUSSION}

As it would be the case with any magnetograph observation of features A and B (regardless of the instrument used or the spatial resolution), the magnetograms of Figure 2 provide little information on their magnetic structure for two reasons.

First, the observed polarities do not reflect true field inclinations because of projection effects (the sunspot was located $43^{\circ}$ off the disk center). To derive true inclinations one has to infer the vector magnetic field in the LOS frame and transform it to the local reference frame, which can only be done through inversions of all four Stokes spectra.

Second, and more important, abnormal Stokes $V$ profiles with three or four lobes occur frequently in MMFs. This is demonstrated for the first time by our observations. In Figure 3 we show an example of cospatial Stokes $I$ and $V$ profiles of Fe I 630.15, Fe I 630.25, Fe I 1564.85, and Fe I $1565.2 \mathrm{~nm}$ emerging from a negative-polarity pixel inside feature A. The very complex shapes displayed by the Stokes $V$ profiles of the four lines may be caused by strong gradients of the atmospheric parameters along the LOS and/or the presence of several magnetic components in the resolution element. The problem with such abnormal profiles is that the meaning of polarity looses its sense, as both positive and negative polarities can be assigned to the same pixel depending on the exact wavelength(s) used to construct the magnetogram. It is not surprising that the existence of abnormal profiles has gone unnoticed: most of what we know today about MMFs has been learned from magnetograph observations that cannot reveal 


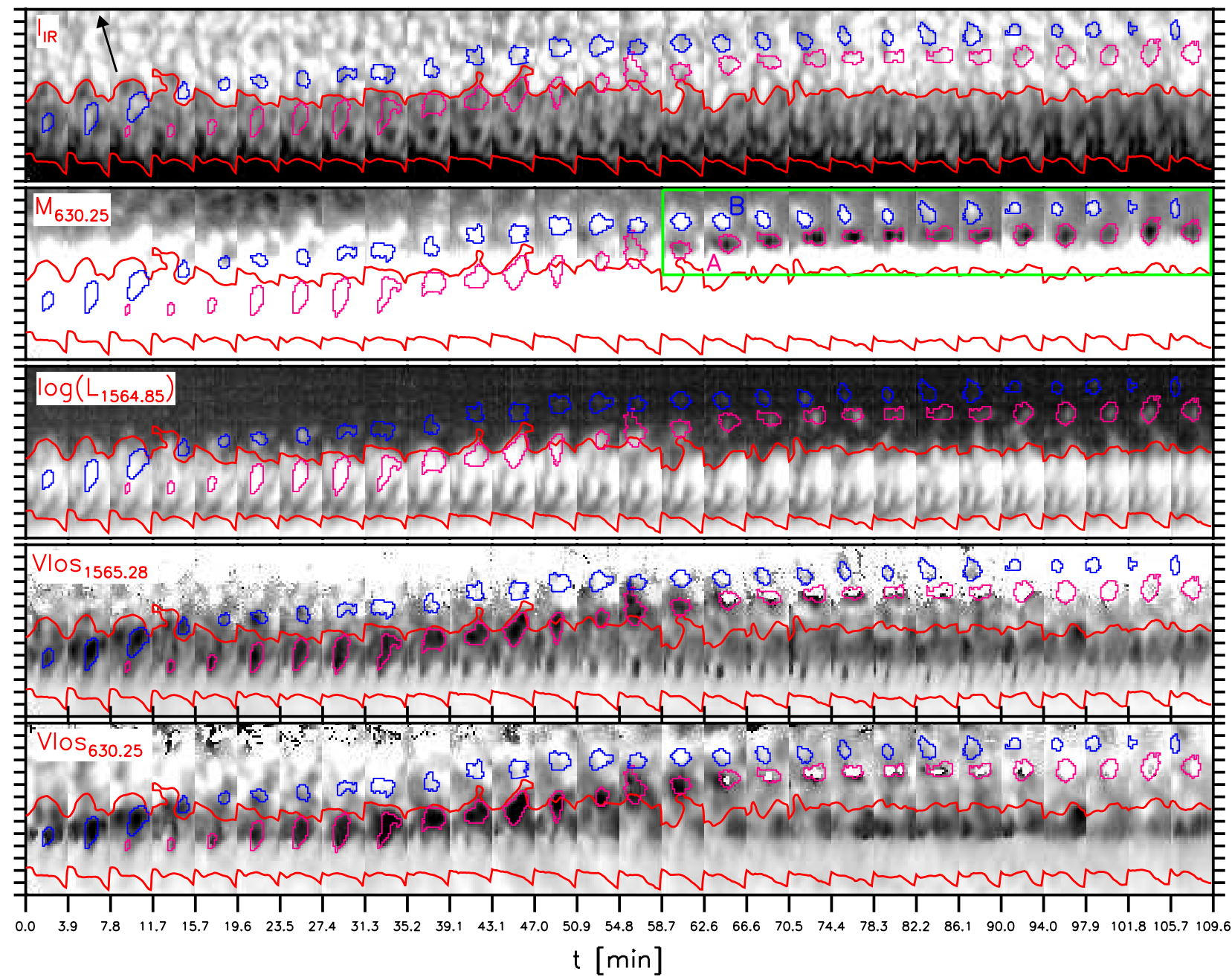

FIG. 2.- From top to bottom: Continuum intensity at $1565 \mathrm{~nm}$, magnetogram signal from Fe I $630.25 \mathrm{~nm}$, logarithm of the total linear polarization of Fe I $1564.85 \mathrm{~nm}$, Stokes $V$ zero-crossing velocity of Fe I $1565.28 \mathrm{~nm}$, and Stokes $V$ zero-crossing velocity of Fe I $630.25 \mathrm{~nm}$. The gray scale ranges from 0.82 to 0.95 in the first panel, from $-0.5 \%$ to $1 \%$ in the second panel, from -0.97 to 0.80 in the third panel, from -4.0 to $0 \mathrm{~km} \mathrm{~s}^{-1}$ in the fourth panel, and from -2.8 to $0 \mathrm{~km} \mathrm{~s}^{-1}$ in the last panel. Negative velocities are blueshifts. Pink and blue contours delimit features A and B, respectively. Red lines indicate the inner and outer penumbral boundaries. Each tickmark in the $y$-axis represents $1^{\prime \prime}$. The arrow marks the direction toward disk center. $t=0$ minutes corresponds to June $30,9: 34$ UT.

this kind of spectral subtleties. One should realize, however, that gross errors may result from direct interpretations of magnetograms when abnormal profiles exist, simply because even the polarity of the field would be an ill-defined quantity. This is the reason why we refrain from attempting a definite classification of the observed MMFs. The conclusion that MMFs can be traced back to Evershed clouds appearing in the midpenumbra, however, is a solid result that does not depend in any way on the exact nature of the flux concentrations.

\section{CONCLUSIONS}

We have used simultaneous spectropolarimetric measurements in two spectral regions to demonstrate the relation between the penumbral Evershed flow and moving magnetic features (MMFs). Our observations combine high cadence (3.9 minutes) with one of the highest spatial resolutions achieved in solar spectropolarimetry $(\sim 0$ !'7).

One after the other, two Evershed clouds are seen to move radially outward along a penumbral filament characterized by larger field inclinations to the LOS than its surroundings. They appear in the center-side penumbra, exhibiting blueshifts and positive polarity (i.e., the polarity of the spot). Eventually, the clouds cross the outer penumbral boundary and become MMFs. Once in the moat region, the flux patch closer to the spot shows negative polarity. The two MMFs are separated by about $2^{\prime \prime}$ and move away from the spot with an average speed of $0.5 \mathrm{~km} \mathrm{~s}^{-1}$. We have examples of other Evershed clouds crossing the outer penumbral boundary and becoming MMFs, so the process just described may be quite common in sunspots.

Our observations demonstrate that at least some MMFs are a manifestation of the penumbral Evershed flow in the sunspot moat. This, in turn, gives support to earlier results about the magnetic connection between MMFs and the penumbra (e.g., Zhang et al. 2003; Sainz Dalda \& Martínez Pillet 2005). The association between Evershed clouds and MMFs found here suggests that the physical structures responsible for the existence of the former inside the penumbra are also present in, or propagate to, the sunspot moat. This may help refine theoretical models of sunspot penumbrae.

We have also shown, for the first time, that abnormal circular polarization profiles with three or four lobes occur in MMFs. The presence of abnormal profiles hampers direct interpretations of magnetogram polarities in terms of magnetic field inclinations. As a result, we do not know if the vector magnetic field is really pointing downward in the negative- 

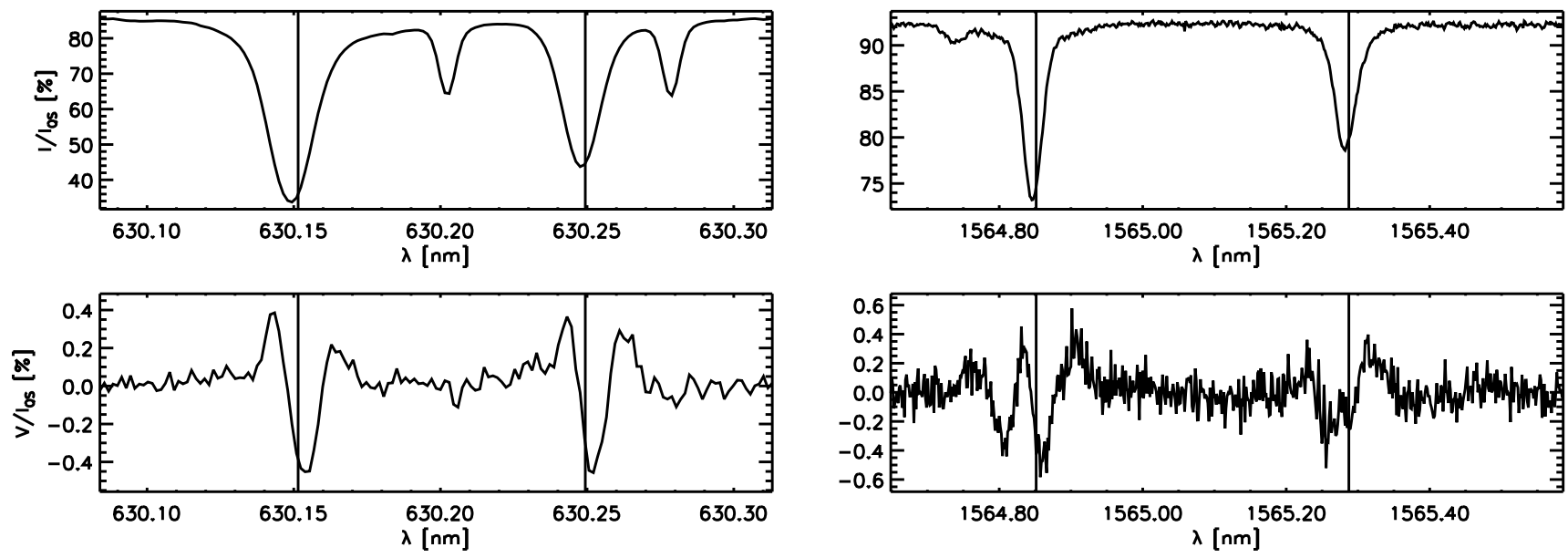

FIG. 3.- Cospatial Stokes I (top) and V (bottom) profiles of Fe I 630.15, Fe I 630.25, Fe I 1564.85, and Fe I 1565.2 emerging from a pixel inside feature A with abnormal circular polarization signals. The vertical solid lines represent the rest wavelengths of the spectral lines.

polarity MMF and upward in the the positive-polarity MMF, or if the field azimuth provides any evidence of magnetic connection between the two flux concentrations. To investigate these issues, our efforts will concentrate on the inversion of the observed Stokes spectra.

Discussions with J.M. Borrero and A. Sainz Dalda are gratefully acknowledged. The DOT G-band image was taken from http://dotdb.phys.uu.nl/DOT/ This work has been supported by the Spanish MEC through Programa Ramón y Cajal and projects ESP2002-04256-C04-01 and ESP2003-07735C04-03, and by the German DFG through grant SCHL 514. The VTT is operated by the Kiepenheuer-Institut für Sonnenphysik at the Spanish Observatorio del Teide of the Instituto de Astrofísica de Canarias.

\section{REFERENCES}

Beck, C., Schmidt, W., Kentischer, T., \& Elmore, D. 2005a, A\&A, 437, 1159 Beck, C., Schlichenmaier, R., Collados, M., Bellot Rubio, L., \& Kentischer, T. 2005b, A\&A, 443, 1047

Beck, C., Bellot Rubio L. R., Schlichenmaier, R., \& Sütterlin P. 2006, A\&A, submitted

Bonet, J.A., Márquez, I., Muller, R., Sobotka, M., \& Tritschler, A. 2004, A\&A, 423, 737

Borrero, J.M., \& Bellot Rubio, L.R. 2002, A\&A, 385, 1056

Harvey, K., \& Harvey, J. 1973, Sol. Phys., 28, 61

Kubo, M. 2005, Ph.D. thesis, Univ. Tokyo

Lee, J.W. 1992, Sol. Phys., 139, 267

Martínez Pillet, V., et al. 1999, ASP Conf. Ser. 183, High Resolution Solar Physics: Theory, Observations, and Techniques, ed. T.R. Rimmele, K.S. Balasubramaniam, \& R.R. Radick (San Francisco: ASP), 264

Martínez Pillet, V., Lites, B.W., \& Skumanich, A. 1997, ApJ, 474, 810

Martínez Pillet, V. 2002, Astron, Nachr., 323, 342

Nye, A.H., Thomas, J.H., Cram, L.E. 1984, ApJ, 285, 381

Penn M.J., Jaeggli, S.A., Henney, C.J., Walton, S.R., \& Luszcz, S.H. 2006, ASP Conf. Ser., Solar Polarization Workshop 4, ed. R. Casini \& B.W. Lites (San Francisco: ASP), in press
Rimmele, T.R. 1994, A\&A, 290, 972

Rouppe van der Voort, L.H.M. 2003, A\&A, 397, 757

Sainz Dalda, A., \& Martínez Pillet, V. 2005, ApJ, 632, 1176

Schlichenmaier, R. 2002, Astron. Nachr., 323, 303

Sheeley, N.R. 1969, Sol. Phys., 9, 347

Solanki, S.K. 2003, A\&A Rev., 11, 153

Soltau, D., Berkefeld, T., von der Lühe, O., Wöger, F., \& Schelenz, T. 2002 Astron. Nachr., 323, 236

Shine, R.A., Title, A.M., Tarbell, T.D., Smith, K., Frank, Z.A., \& Scharmer, G. 1994, ApJ, 430, 413

Shine, R., \& Title, A.M. 2001, in Encyclopedia of Astronomy and Astrophysics, ed. P. Murdin (Bristol: IOP), 3209

Thomas, J.H., Weiss, N.O., Tobias, S.M., \& Brummell, N.H. 2002, Nature, 420, 390

Yurchyshyn, V.B., Wang, H., \& Goode, P.R. 2001, ApJ, 550, 470

Zhang, J., Solanki, S.K., \& Wang, J. 2003, A\&A, 399, 755 